\title{
Highly cited papers on tuberculosis (2003-2005)
}

The following tables show the primary research papers on tuberculosis published between 2003 and 2005 that have had the highest number of citations in the literature. To create these tables, we queried the Scopus database (http://www.scopus.com) to search for articles that included the term 'tuberculosis' in the title, abstract or keywords. After sorting the results on the basis of citation number, we removed review articles, as well as clinical trials, epidemiological, diagnostic and methodological papers, and studies on forms of tuberculosis that affect cattle and other species. The number of citations is accurate as of 12 February 2007. The tables include every paper that has been cited at least 100, 50 and 30 times (2003, 2004 and 2005 tables, respectively).

Highly cited research papers on tuberculosis published in 2003

Reference

Times cited

Geijtenbeek, T.B. et al. Mycobacteria target DC-SIGN to suppress dendritic cell function. J. Exp. Med. 197, 7-17. 236

Sassetti, C.M. et al. Genes required for mycobacterial growth defined by high density mutagenesis. Mol. Microbiol. 48, 77-84.

Garnier, T. et al. The complete genome sequence of Mycobacterium bovis. Proc. Natl. Acad. Sci. USA 100, 7877-7882.

Tallieux, L. et al. DC-SIGN is the major Mycobacterium tuberculosis receptor on human dendritic cells. J. Exp. Med. 197, $121-127$.

Pym, A.S. et al. Recombinant BCG exporting ESAT-6 confers enhanced protection against tuberculosis. Nat. Med. 9, 533-539.

Schnappinger, D. et al. Transcriptional adaptation of Mycobacterium tuberculosis within macrophages: insights into the phagosomal 140 environment. J. Exp. Med. 197, 693-704.

Voskuil, M.I. et al. Inhibition of respiration by nitric oxide induces a Mycobacterium tuberculosis dormancy program. J. Exp. Med. 198,

705-713.

\section{Highly cited research papers on tuberculosis published in 2004}

\section{Reference}

McShane, $\mathrm{H}$. et al. Recombinant modified vaccinia virus Ankara expressing antigen 85A boosts BCG-primed and naturally acquired

Times cited antimycobacterial immunity in humans. Nat. Med. 10, 1240-1244.

Reed, M.B. et al. A glycolipid of hypervirulent tuberculosis strains that inhibits the innate immune response. Nature 430, 84-87.

Gutierrez, M.G. et al. Autophagy is a defense mechanism inhibiting BCG and Mycobacterium tuberculosis survival in infected macrophages Cell 119, 753-766.

Skeiky, Y.A. et al. Differential immune responses and protective efficacy induced by components of a tuberculosis polyprotein vaccine,

Mtb72F, delivered as naked DNA or recombinant protein. J. Immunol. 172, 7618-7628.

Tsolaki, A.G. et al. Functional and evolutionary genomics of Mycobacterium tuberculosis: insights from genomic deletions in 100 strains. Proc. Natl. Acad. Sci. USA 101, 4865-4870.

Guinn, K.M. et al. Individual RD1-region genes are required for export of ESAT-6/CFP-10 and for virulence of Mycobacterium tuberculosis. Mol. Microbiol. 51, 359-370.

Schmidt, F. et al. Complementary analysis of the Mycobacterium tuberculosis proteome by two-dimensional electrophoresis and isotopecoded affinity tag technology. Mol. Cell. Proteomics. 3, 24-42.

\section{Highly cited research papers on tuberculosis published in 2005}

Andries, K. et al. A diarylquinoline drug active on the ATP synthase of Mycobacterium tuberculosis. Science 307, 223-227.

Muñoz-Elias, E.J. \& McKinney, J.D. Mycobacterium tuberculosis isocitrate lyases 1 and 2 are jointly required for in vivo growth and virulence. Nat. Med. 11, 638-644.

Pan H. et al. Ipr1 gene mediates innate immunity to tuberculosis. Nature 434, 767-772.

Langermans, J.A. et al. Protection of macaques against Mycobacterium tuberculosis infection by a subunit vaccine based on a fusion protein of antigen 85B and ESAT-6. Vaccine 23, 2740-2750.

Grode, L. et al. Increased vaccine efficacy against tuberculosis of recombinant Mycobacterium bovis bacille Calmette-Guerin mutants that secrete listeriolysin. J. Clin. Invest. 115, 2472-2479.

Vergne, I. et al. Mechanism of phagolysosome biogenesis block by viable Mycobacterium tuberculosis. Proc. Natl. Acad. Sci. USA 102 4033-4038. 